\title{
Reconstrucción en un tiempo del pabellón auricular en amputación parcial traumática con preservación del pedículo inferior: Revisión de la literatura y presentación de dos casos
}

\section{One-stage auricular replantation in traumatic partial ear amputation with inferior pedicle preservation: Review of the literature and presentation of two cases}

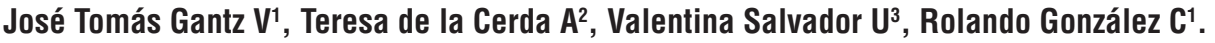

\begin{abstract}
RESUMEN
La amputación parcial traumática del pabellón auricular con preservación del pedículo inferior corresponde a una patología infrecuente. Su reconstrucción incluye el uso de diversas técnicas quirúrgicas. Presentamos dos casos de reimplante inmediato, sin uso de microcirugía, con resultados favorables y sin necesidad de reintervenciones, junto a una revisión de la literatura. La revisión bibliográfica realizada apoya el uso de esta técnica, basándose en la preservación del puente cutáneo bajo el tragus que incluye una rama de la arteria temporal superficial y que sería responsable de la irrigación del reimplante.
\end{abstract}

Palabras clave: Oreja, amputación traumática, reimplante.

\begin{abstract}
Partial auricle amputation with preservation of the inferior pedicle is an unusual pathology. The reconstruction in these cases includes many surgical techniques. We present two cases of immediate replantation, without the use of microsurgery, with favorable outcomes and without need of new interventions. The revision of the literature supports this technique based in the preservation of the cutaneus bridge under the tragus that includes a branch of the superficial temporal artery that is responsible of the perfusion of the reimplant.
\end{abstract}

Key words: Ear, amputation traumatic, replantation.

\section{INTRODUCCIÓN}

La información respecto de la amputación parcial del pabellón auricular reportada en la literatura hace referencia sólo a reportes de casos aislados ${ }^{1}$, con resultados variables y sin la posibilidad de realizar análisis estadísticos de los mismos. El reimplante del pabellón auricular completamente amputado puede requerir diferentes procedimientos para realizarse, tanto en forma inmediata como diferida.

\footnotetext{
${ }^{1}$ Cirujano General. Servicio de Cirugía Hospital Padre Hurtado, Santiago. Chile.

${ }^{2}$ Cirujano Plástico. Centro Cirugía Estética, Santiago. Chile.

${ }^{3}$ Residente Programa de Cirugía General Adulto, Clínica Alemana Universidad del Desarrollo.
} 
Entre las diferentes alternativas de reconstrucción de éste podemos mencionar desde el uso de técnicas microquirúrgicas, como una herramienta fundamental, hasta técnicas reconstructivas del pabellón auricular externo con preservación del esqueleto cartilaginoso o el uso de injertos de cartílago y material protésico para lograr buenos resultados funcionales y estéticos. Tal como mencionamos previamente, las amputaciones parciales del pabellónauricular son anecdóticamente reportadas en la literatura ${ }^{2,3}$, sobre todo aquellas en las que el pedículo remanente es el inferior (bajo el tragus). En estas últimas, la posibilidad de supervivencia del pabellón auricular, depende de la indemnidad de un puente cutáneo que contenga su vascularización ${ }^{1,5}$.

El presente trabajo, presenta dos casos de amputación parcial de pabellón auricular así como la revisión bibliográfica de las amputaciones parciales del pabellón auricular externo.

Realizamos una revisión de registros clínicos de pacientes operados de urgencia en el Hospital Padre Hurtado con diagnóstico de "amputación parcial traumática del pabellón auricular" desde el 1 de enero de 2010 hasta el 30 de diciembre de 2014. Se verificó en el examen físico de ingreso y en el protocolo operatorio que existiera indemnidad del pedículo inferior y que el procedimiento quirúrgico correspondiera a reimplante primario, sin uso de microcirugía. Se excluyeron pacientes sometidos a otros procedimientos como retoques o cirugía electiva. Los datos fueron obtenidos de la ficha electrónica de urgencia y protocolos operatorios. Los pacientes autorizaron el uso de dicha información con fines académicos. Se realizó un seguimiento durante un año en controles ambulatorios, realizándose una evaluación clínica del reimplante, registro fotográfico y una evaluación de satisfacción del paciente, que incluyó tanto el resultado funcional como estético.

La revisión bibliográfica se realizó en PUBMED e incluyó artículos hasta el 30 de diciembre de 2014, usando los términos: "Ear subtotal amputation / replantation" y "Ear partial amputation / replantation". Se limitó la búsqueda a publicaciones en inglés o español. Se excluyeron aquellos trabajos que correspondieran a reparación o retoques del pabellón auricular y/o el uso de técnicas microquirúrgicas.

\section{CASOS CLÍNICOS}

\section{Caso 1}

Hombre de 31 años, sano. Presenta herida cortante por vidrio ocurrida 30 minutos previo al ingreso. Al examen físico destaca: sección parcial del pabellón auricular izquierdo y completa del conducto auditivo externo (CAE), respetando el lóbulo $(2,5 \mathrm{~cm}$ de ancho), desde el antitragus hacia distal. Es llevado a pabellón 70 minutos después del ingreso. Se administraron tres dosis de cefazolina 1 gramo ev como profilaxis. Se realizó aseo quirúrgico y regularización de bordes. Se reparó el esqueleto cartilaginoso con sutura discontinua no reabsorbible monofilamento, obteniendo un resultado similar en proyección, posición y tamaño al contralateral. Se suturó con puntos discontinuos de material reabsorbible el pabellón a la fascia temporal. La piel fue suturada con puntos separados de material no reabsorbible. La duración de la cirugía fue de 75 minutos. No se utilizaron antibióticos en el posoperatorio. El paciente es dado de alta al quinto día posoperatorio, sin presentar zonas de sufrimiento o infección ni requerir procedimientos adicionales. El seguimiento al año no muestra defectos del reimplante, el paciente refiere $100 \%$ de satisfacción con el resultado y no presenta disminución de la audición en forma subjetiva (Figuras 1, 2 y 3).

\section{Caso 2}

Hombre de 46 años, hipertenso en tratamiento. Mientras viajaba en automóvil es impactado por el costado por otro vehículo, provocando golpe directo contra la ventana. Consultó al servicio de urgencias 60 minutos posaccidente. Al examen físico de ingreso destaca amputación parcial del pabellón auricular derecho, con indemnidad del CAE y el lóbulo (3 $\mathrm{cm}$ de diámetro), pero con sección completa del hélix y antihélix. El paciente es llevado a pabellón 60 minutos después del ingreso. Se administraron tres dosis de cefazolina 1 gramo ev como profilaxis. Se realizó aseo quirúrgico y regularización de bordes. Se reconstruyó el esqueleto cartilaginoso con sutura discontinua no reabsorbible y se suturó con puntos discontinuos de material reabsorbible 3.0 el pabellón a la fascia temporal. La piel fue suturada con puntos separados 


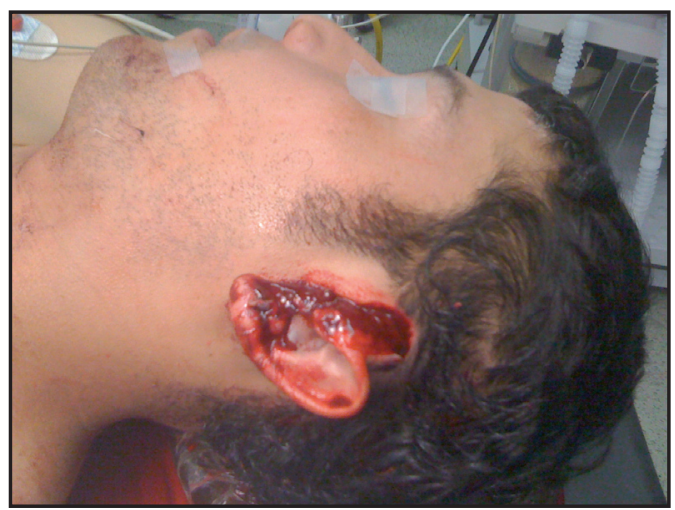

Figura 1. Fotografía preoperatoria paciente 1.

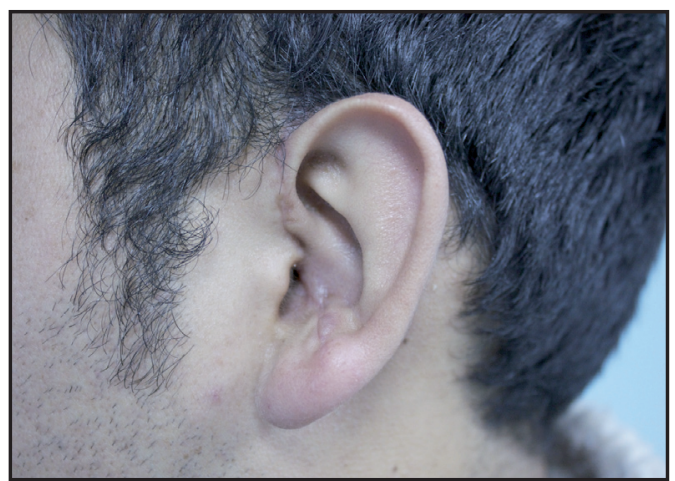

Figura 3. Fotografía control 1 año posoperatorio paciente 1.

de material no reabsorbible. La duración de la cirugía fue de 139 minutos. El paciente es dado de alta al sexto día posoperatorio, sin presentar zonas de sufrimiento o infección ni requerir procedimientos adicionales. El seguimiento al año no muestra defectos del reimplante, el paciente presenta $100 \%$ de satisfacción con el resultado y no refiere disminución subjetiva de la audición (Figuras 4, 5 y 6).

El resultado de la búsqueda en PUBMED arrojó un total de 30 publicaciones. Después de aplicar los criterios de exclusión, quedarían seleccionadas sólo 5. Cuatro corresponden a reportes de casos y una a revisión de la anatomía del pabellón auricular realizada en cadáveres; las cuales corresponden a nuestra revisión.

El estudio de Tomono ${ }^{1,3}$ es la serie que cuenta con más casos, reportando dos pacientes. El

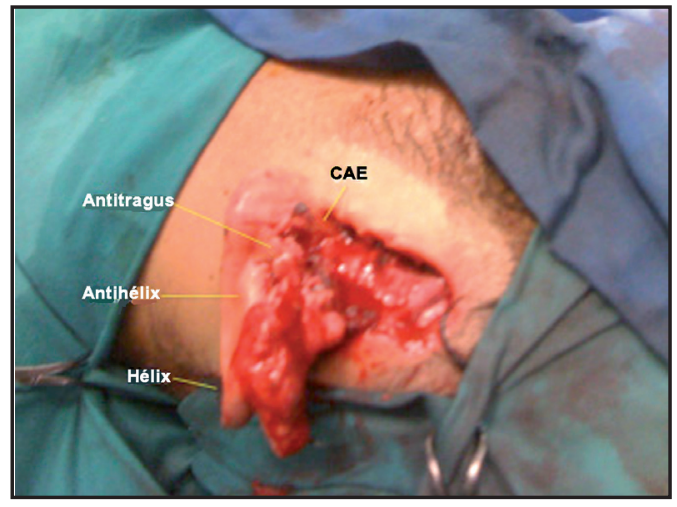

Figura 2. Fotografía preoperatoria paciente 1.

primer paciente de dicha serie presentó un lóbulo indemne de $1,5 \times 3 \mathrm{~cm}$. Se realizó reimplante inmediato y se indicó heparinización y la administración de un preparado con nicotina posoperatoria. El paciente evoluciona con un marcado edema del pabellón auricular y necrosis de la línea de sutura y parte de la piel y cartílago de la concha. El segundo caso presentó un lóbulo de $1 \mathrm{~cm}$, recibió un tratamiento similar al ya descrito y evolucionó con necrosis de la línea de sutura, sin otras complicaciones. Clodius ${ }^{1,2}$ presenta dos casos de reimplantes de los cuales sólo uno corresponde a la preservación del pedículo inferior; el cual midió $3,5 \mathrm{~cm}$ de diámetro. Se realizó reimplante inmediato, sin complicaciones posoperatorias. Ozçelik ${ }^{1}$ y cols, presentan un paciente masculino de 36 años de edad con amputación subtotal de pabellón auricular derecho tras sufrir un accidente de tránsito. El pedículo indemne correspondía al superior, con un puente cutáneo que no superaba los $6 \mathrm{~mm}$. Se realizó reimplante inmediato, sin uso de técnicas microquirúrgicas. Se administró dextran 40 durante 5 días. No reporta complicaciones en el posoperatorio inmediato, logrando adecuada proyección y contorno del reimplante. Safak ${ }^{4}$ presenta un único caso, mujer de 40 años de edad que tras haber sufrido accidente en carretera de alta velocidad, presentó la amputación subtotal del pabellón auricular derecho con pedículo cutáneo superior de $3 \mathrm{~mm}$. Se realizó implante inmediato del mismo, sin el uso de microcirugía. En dicho reporte se menciona el sufrimiento y posterior pérdida del lóbulo del pabellón implantado, el que debió ser reconstruido 


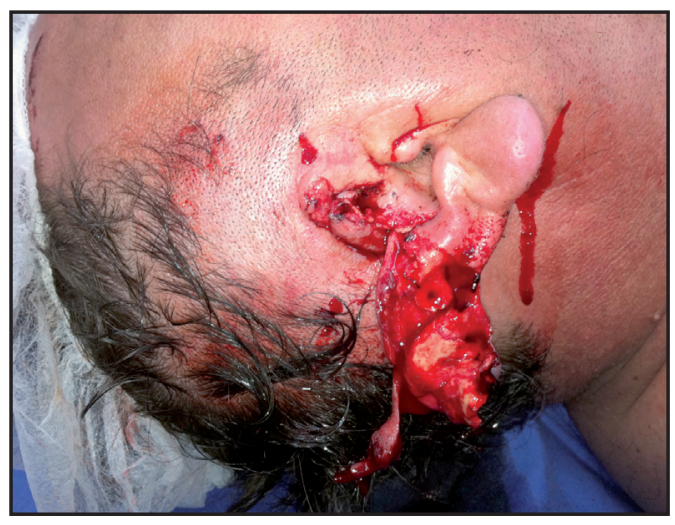

Figura 4. Fotografía preoperatoria paciente 2.

en un segundo tiempo quirúrgico, mediante el uso de colgajos locales. La última de las publicaciones destacadas en la revisión, tiene que ver con el detallado anatómico que realiza Nicolay ${ }^{6}$ de la irrigación del pabellón auricular. A través del estudio de 13 cadáveres, por medio de tinción de látex logra determinar las principales arcadas arteriales encargadas de la irrigación del mismo.

\section{DISCUSIÓN}

La amputación parcial del pabellón auricular es una lesión infrecuente existiendo escasas publicaciones internacionales y ninguna en nuestro medio. Los reportes de reconstrucción del pabellón auricular basados en el pedículo inferior, sin uso de microcirugía ni colgajos adicionales son, por lo anteriormente expuesto, escasos; no existiendo publicaciones con más de dos casos. La sobrevida del reimplante en dichas condiciones ha sido considerada por varios autores como variable, desde necrosis de la línea de sutura hasta necrosis parcial de reimplante.

Nicolay describe la irrigación del pabellón auricular externo mediante la inyección de látex en trece cadáveres. Por este método demuestra la presencia de una pequeña rama superior de la arteria superficial temporal por sobre el tragus, la que se extiende a lo largo del reborde superior de la aurícula y se une con la arcada helicoidal. Inferior al tragus, se describe una segunda rama horizontal de la arteria superficial temporal5,6. Dicho

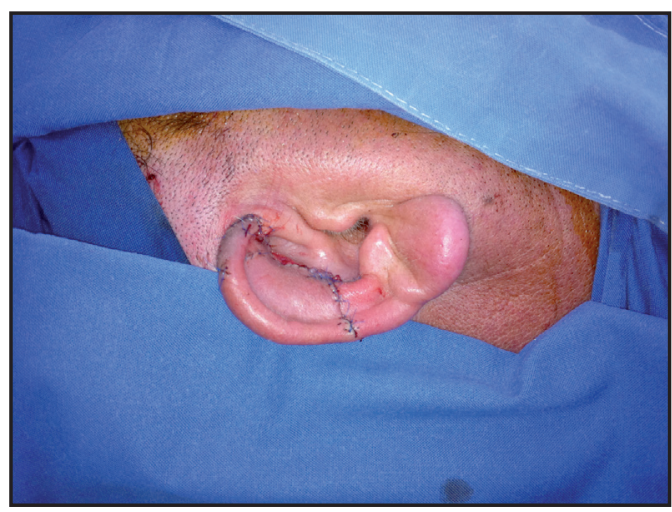

Figura 5. Fotografía posoperatoria inmediata paciente 2.

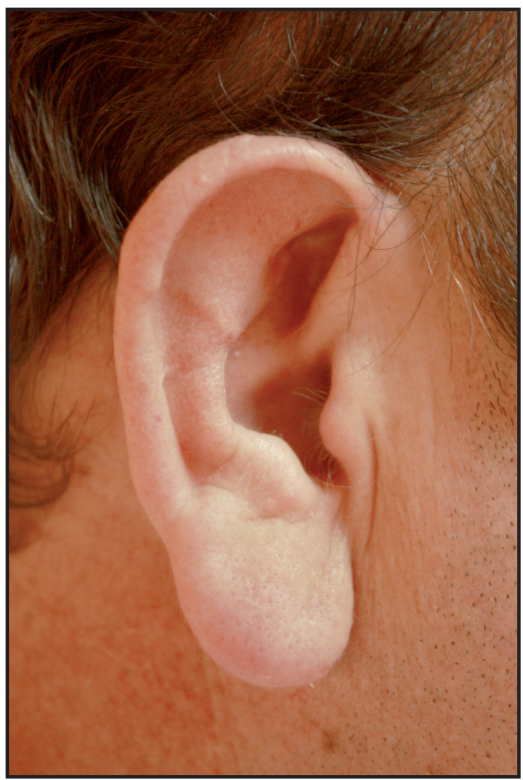

Figura 6. Fotografía control 1 año posoperatorio paciente 2.

estudio concluye que la aurícula puede sobrevivir en una amputación parcial basando su irrigación en sólo una de las ramas de la arteria temporal superficial.

Yotsuyanagi ${ }^{1}$ plantea que los mayores determinantes que definirán el reimplante inmediato del pabellón auricular o la necesidad de realizar anastomosis microquirúrgicas serían la relación entre el segmento remanente y la vasculatura, el 
grado de congestión auricular y el grado de sangrado del muñón. De no preservarse el pedículo vascular, deben considerarse la microcirugía y los procedimientos de rescate del esqueleto cartilaginoso como únicas alternativas de supervivencia del pabellón auricular.

En nuestro estudio presentamos dos casos en los cuales se preservó un puente cutáneo de 2,5 y $3 \mathrm{~cm}$, los cuales fueron suficientes para preservar la rama de la arteria temporal superficial y así irrigar correctamente el pabellón reimplantado.

\section{CONCLUSIÓN}

La escasa casuística no permite el análisis estadístico de esta patología, sin embargo podemos plantear que el reimplante inmediato del pabellón auricular parcialmente amputado con preservación del pedículo inferior, sin necesidad de microcirugía u otros procedimientos adicionales, puede ser exitoso. Este resultado favorable estaría basado en una rama horizontal de la arteria temporal superficial que se ubica de manera constante bajo el tragus y que, de mantenerse indemne, podría mantener una adecuada perfusión.

\section{BIBLIOGRAFÍA}

1. Ozçelik D, Unveren T, Toplu G. Subtotal ear amputation with a very narrow pedicle: a case report and review of the literature. Ulus Travma Acil Cerrahi Derg 2009; 15(3): 306-10.

2. Clodius L. Local hypothermia for the avulsed external ear. Br J Plast Surg 1968; 21: 250-2.

3. Tomono T, Hirose T. Treatment of the subtotally amputated auricle. Jpn J Plast Reconstr Surg 1980; 23: 41-6.

4. SAFAK T, KaYIKCIOGLU A. A traumatic ear amputation attached with a narrow pedicle. Ann Plast Surg 1998; 40: 106-7.

5. BRENT B. Reconstruction of the auricle. In: McCarthy JG, editor. Plastic surgery. Vol 3, Philadelphia: W.B. Saunders; 1990. p. 2094-152.

6. Nicolay NH, Bruno AD, Follmar KE, Stokes th, Gonyon DL, Marcus JR, et all. Near-total ear amputation: anatomical bases and management. Chirurg 2007; 78(9): 835-9.

Dirección: José Tomás Gantz V. 